\title{
Characterization of ALPIDE silicon sensors with inclined tracks
}

\section{S. Kushpil* for the ALICE Collaboration}

Nuclear Physics Institute of the Czech Academy of Sciences, Rez, Czechia

\begin{abstract}
The upgrade of the ALICE Inner Tracking System (ITS) aims at improving the capabilities of ALICE in terms of read-out rate as well as track pointing resolution and track finding efficiency, especially for particles with low transverse momenta. The new ITS will be a low material budget detector with high granularity and read-out speed. It comprises seven concentric layers of Monolithic Active Pixel Sensors (MAPS) with a total active surface of about $10 \mathrm{~m}^{2}$. The developed MAPS are based on the TowerJazz $180 \mathrm{~nm}$ CMOS technology. The sensor is called ALPIDE. In this paper we present the setup used for measurements with inclined tracks and we discuss the sensor efficiency obtained using $\pi$ beams with a momentum of $6 \mathrm{GeV} / \mathrm{c}$ at the Proton Synchrotron (PS) at CERN. Some sensors were irradiated before the beam test using the cyclotron facility of the Nuclear Physics Institute of the Czech Academy of Sciences (NPI CAS) to induce radation damage to the sensor. Measurements at different operating points (thresholds, bias voltages) provide important information about cluster-shape frequencies, needed to tune the ALICE Monte-Carlo generators. Very good agreement between test-beam data and simulations is obtained.
\end{abstract}

International Conference on High Energy Physics - ICHEP2020-

28 July - 6 August, 2020

Prague, Czech Republic

*Speaker. 


\section{General ALICE upgrade}

ALICE [1] is a large-scale, heavy-ion physics experiment at the CERN Large Hadron Collider (LHC). It is designed to study properties of the Quark-Gluon Plasma (QGP) using nucleusnucleus, proton-nucleus, and proton-proton collisions.

After the second long shutdown of the LHC, ALICE will focus on high-precision measurements of heavy-flavor hadrons, quarkonia, and low-mass dileptons at low transverse momenta [2]. ALICE will run in a continuous read-out mode and it will collect minimum bias $\mathrm{Pb}-\mathrm{Pb}$ events. In LHC Run 3 and Run 4, ALICE subdetectors will have to be able to handle the read-out at a Pb-Pb collision rate of $100 \mathrm{kHz}$. The upgraded detector will record an integrated luminosity of more than $10 \mathrm{nb}^{-1}$ in $\mathrm{Pb}-\mathrm{Pb}$ collisions which means a factor 100 more when compared to the data that he been recorded up to 2018. The ALICE upgrade strategy includes several projects [2]. Two new detectors based on the ALPIDE chip will be installed. The first detector will be a high resolution, low material budget ITS that will replace the present ITS. The second detector will be the Muon Forward Telescope [3], a silicon tracker placed in front of the muon spectrometer.

\section{The new Inner Tracking System}

The goals of the ITS upgrade [4] are to improve the impact parameter resolution (by a factor of 5 in the $z$ direction and by a factor of 3 in the bending $r \varphi$ direction) and to improve the tracking efficiency and the transverse momentum resolution at very low $p_{\mathrm{T}}(<1 \mathrm{GeV} / c)$. This will be achieved by placing the first layer of silicon detectors closer to the interaction point. The reduction of the beam pipe diameter in the center of ALICE will allow the first ITS layer to be moved as close as $23 \mathrm{~mm}$ to the interaction point instead of the original $39 \mathrm{~mm}$. The impact parameter resolution and the tracking performance will gain from reduction in the material budget, in particular in the innermost layers. The use of Monolitic Active Pixel Sensors, the optimization of the front-end electronics, and the consequent reduction of the power and signal cables will reduce the total material budget $X / X_{0}$ per layer from the present $1.14 \%$ to $0.3 \%$ for the three innermost layers and to about $1 \%$ for the outer layers. The new ITS will consist of seven layers of silicon pixel sensors which will cover an area of about $10 \mathrm{~m}^{2}$ and which will consist of about $1.25 \times 10^{10}$ pixels. The same ALPIDE sensor, whose pixel size is $27 \mu \mathrm{m} \times 29 \mu \mathrm{m}$, will be used to equip all layers. Let us note that the first ALICE ITS had six layers of silicon sensors and its Silicon Pixel Detector had a pixel size of $50 \mu \mathrm{m} \times 425 \mu \mathrm{m}$. The seven layers of the new ITS will be grouped as follows: the three innermost layers will make up the Inner Barrel and the four outermost layers the Outer Barrel. The layers will be segmented in $r \varphi$ direction into elements called staves, which extend to the full length of the detector in the direction of the beam. The new design allows fast removal of the detector for maintenance and replacement of faulty modules which can take place, e.g. during yearly shutdowns.

The first ITS runs up to a maximum read-out rate of $1 \mathrm{kHz}$. The upgraded ITS aims to read out data up to a rate of $100 \mathrm{kHz}$ in $\mathrm{Pb}-\mathrm{Pb}$ collisions and $1 \mathrm{MHz}$ in pp collisions. For what concerns the environment in which the new ITS will operate, simulations have shown that the overall dose expected for the innermost layer can reach up to a total ionizing dose (TID) of $270 \mathrm{krad}$ and an 
accumulated non-ionizing energy loss (NIEL) of $1.7 \times 10^{12} 1 \mathrm{MeV} \mathrm{n}_{\mathrm{eq}} \mathrm{cm}^{-2}$ for the full integrated luminosity over the lifetime of the detector [4].

\section{ALPIDE pixel sensors}

ITS sensors have to fulfill the project requirements in terms of granularity, material budget, read-out speed, power consumption and radiation hardness [4]. The $180 \mathrm{~nm}$ CMOS technology by TowerJazz has been selected for the implementation of the pixel chip for all layers of the new ITS. The chosen feature size and the number of metal layers available are adequate to implement high density, low power digital circuits. For the development of the ALPIDE sensor, it was important that this technology allows the production of chips on wafers with high resistivity $(>1 \mathrm{k} \Omega \mathrm{cm})$ [5] and the implementation of an epitaxial layer with thickness greater than $18 \mu \mathrm{m}$ [6]. The final design of the ALPIDE for the new ITS has $25 \mu \mathrm{m}$ thick epitaxial layer. The ALPIDE has $512 \times 1024$ digital pixels with the size of $27 \mu \mathrm{m} \times 29 \mu \mathrm{m}$ that are read out in a binary (hit/no-hit) fashion. This pixel pitch makes it possible to achieve resolution better than $5 \mu \mathrm{m}$. According to the measurements of the chip supply current, the average power density across the surface of the sensor is less than $40 \mathrm{~mW} \mathrm{~cm}^{-2}$.

\section{ALPIDE performance in test beams with inclined tracks}

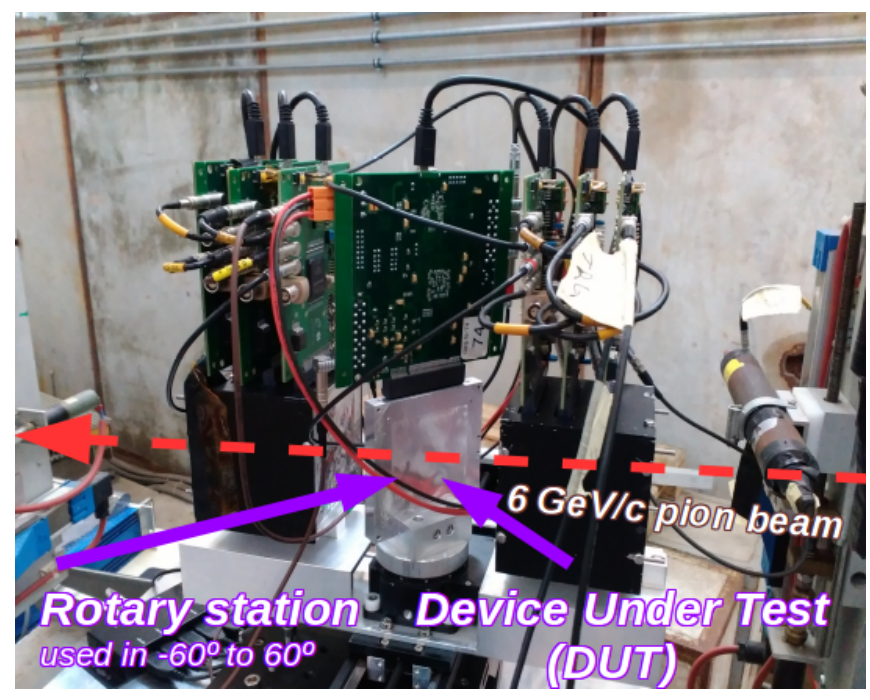

Figure 1: The ALPIDE test beam telescope set-up used for data taking at different incident angles of the beam on the DUT.

The chip was then characterized with a $6 \mathrm{GeV} / c$ negative pion beam at the CERN PS. The test was carried out using a telescope consisting of 7 planes of ALPIDE sensors. The test beam setup is sketched in Figure 1. The device under test (DUT) is located in the middle of the telescope. A charged particle propagating through the setup will ideally make a hit in every plane. The hit information from the tracking planes is used to reconstruct a trajectory of the incident particle and to find an extrapolated crossing point of the track with the DUT. This point is then compared with the measured hit location in the DUT. Thereby quantities like the position resolution or the detection 
efficiency of the DUT can be measured. This analysis is done using the EUTelescope [8] software framework. The purpose of the test beam at the CERN PS was to compare the performance of the non-irradiated sensors and that of a sensor irradiated before the beam test using the cyclotron facility of the NPI CAS. The U-120M cyclotron in NPI CAS was used for an irradiation of the ALPIDE chip with a $30 \mathrm{MeV}$ proton beam [9] during which the ALPIDE sensor was exposed to a dose of $100 \mathrm{krad}$ (TID) and $1.0 \times 10^{12} 1 \mathrm{MeV} \mathrm{n}_{\mathrm{eq}} \mathrm{cm}^{-2}$ (NIEL). The ITS upgrade technical design report [4] puts constraints on the ALPIDE sensor to have the detection efficiency for MIP better than $99 \%$. This efficiency is achieved for certain range of applied thresholds and inclination angles.

The telescope setup allows us to place the middle sensor on a rotary stage, see Figure 1. This makes it possible to study the ALPIDE chip response to different incident beam angles. The rotary station allowed rotation of the DUT by \pm 60 degrees with respect to the beam direction. In particular, we investigated frequency of different cluster shapes. This measurement is an input to the chip simulation and serves for its benchmark testing.

During this test the reverse substrate bias voltage on the DUT was set to $0 \mathrm{~V}$ or $-3 \mathrm{~V}$. Response of the DUT under different incident beam angles was investigated for several threshold values.

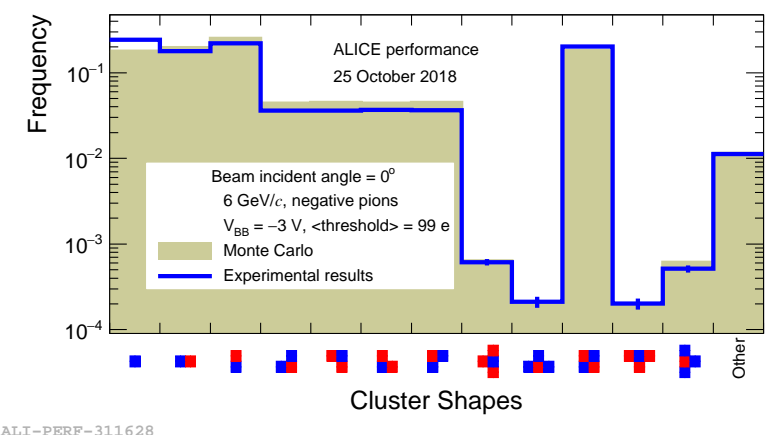

Figure 2: Relative frequency of different cluster shapes in the measurement with a $6 \mathrm{GeV} / c \pi^{-}$beam for a sensor which was perpendicular to the beam direction. The column labeled "other" contains clusters with more than four pixels.

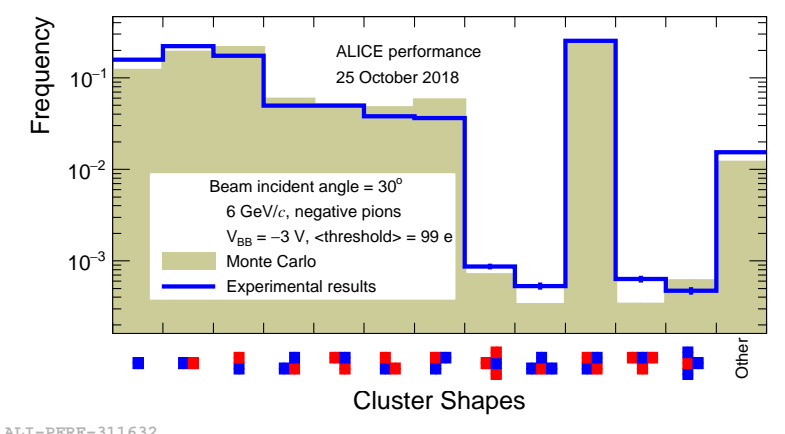

Figure 3: Relative frequency of different cluster shapes in the measurement with a $6 \mathrm{GeV} / c \pi^{-}$beam for a sensor which was inclined by 30 degrees.

Figures 2 and 3 compare relative frequencies of different cluster shapes measured by the non-irradiated sensor when it was oriented perpendicular to the beam and when it was inclined by 30 degrees with respect to the beam direction. The experimental results are marked by a blue 


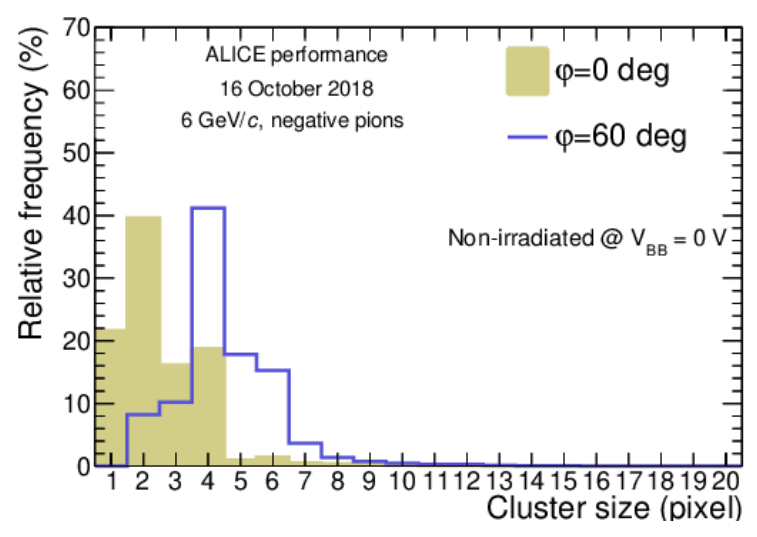

Figure 4: Distribution of cluster size measured for two inclination angles. Data were obtained using a $6 \mathrm{GeV} / c \pi^{-}$beam [7].

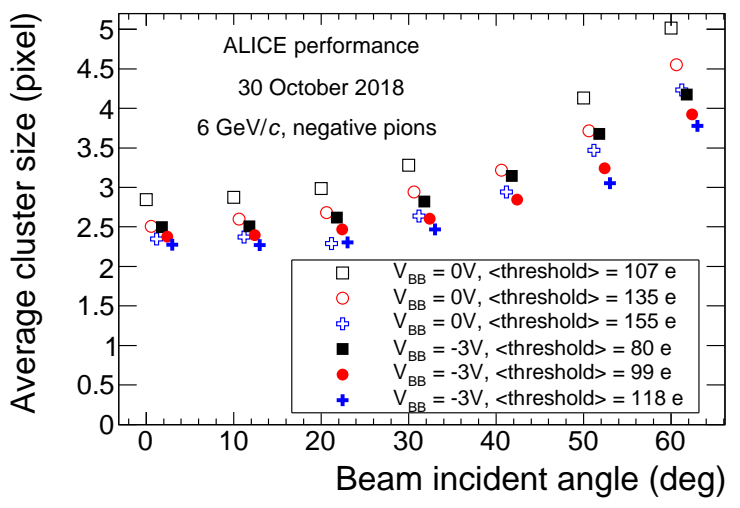

ALI-PERF-311870

Figure 5: The average cluster size as a function of beam incident angle. Data were obtained using a $6 \mathrm{GeV} / c$ $\pi^{-}$beam.

line and they are compared with a simulation which is represented by the brown histogram. The Monte Carlo model of ALPIDE MAPS [10] was developed to simulate diffusion and drift of charge carriers in the electric field of the epitaxial and substrate volumes and takes into account carrier lifetime. The experimental and simulated results show good agreement excepting a tiny part of four pixel clusters of T-shape. As it can be seen from Fig. 4, the relative frequency of one pixel clusters drops to zero when the inclination angle increases to 60 degrees. There is a clear expected tendency to have larger clusters as the inclination angle increases. This trend is confirmed by Figure 5, where we present the average cluster size versus beam incident angle for several threshold values. This test showed that the cluster size decreases with the increase in threshold. The average cluster size is larger in the case of reverse bias voltage $V_{B B}=0 \mathrm{~V}$ in comparison with $V_{B B}=-3 \mathrm{~V}$.

Furthermore, we studied ALPIDE sensor detection efficiency as a function of the beam incident angle as shown in Fig. 6. This measurement was carried out for the non-irradiated sensor as 


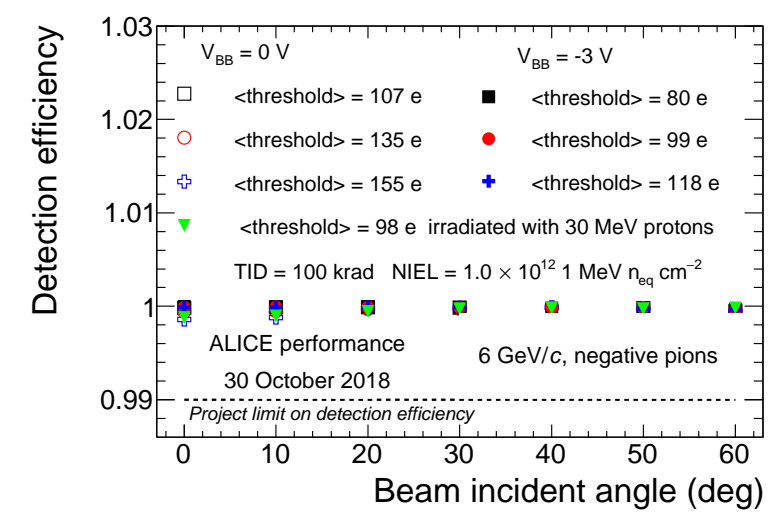

ALI-PERF-311641

Figure 6: Detection efficiency versus beam incident angle obtained using a $6 \mathrm{GeV} / c \pi^{-}$beam.

well as for the sensor irradiated with a TID of $100 \mathrm{krad}$ and a NIEL of $1.0 \times 10^{12} 1 \mathrm{MeV} \mathrm{n}_{\mathrm{eq}} \mathrm{cm}^{-2}$. In all cases, the obtained efficiency meets the project requirement.

\section{Conclusion}

During the LHC Long Shutdown 2 (2019-2022) the ALICE Collaboration will install a new Inner Tracking System based on the ALPIDE pixel sensors. A successful future operation of the newly constructed ITS requires thorough investigations of the ALPIDE sensor performance in beam tests. Here we reported on the performance of the ALPIDE sensor studied with a $6 \mathrm{GeV} / c$ $\pi^{-}$beam. We studied cluster properties and detection efficiency for different incident angles of the beam. The results of our analysis show that the cluster size grows with the increasing inclination angle of the track relative to the sensor normal ("angle of incidence"). The measurement also provides important information about the frequency of occurrence of different cluster shapes. The experimental results discussed in this article serve as an input and a benchmark for simulations of the upgraded ITS.

\section{Acknowledgments}

The work has been supported by the grants LTT17018, LM2015058 of the Ministry of Education of the Czech Republic.

\section{References}

[1] K. Aamodt et al. (ALICE Collaboration) JINST 3 (2008) S08002.

[2] ALICE Collaboration (2012) CERN-LHCC-2012-012, LHCC-I-022.

[3] ALICE Collaboration (2015) CERN-LHCC-2015-001 ALICE-TDR-018.

[4] ALICE Collaboration J. Phys. G41 (2014) 087002, CERN-LHCC-2013-024 ALICE-TDR-017. 
[5] G. Aglieri Rinella, Nucl. Instrum. Meth. A845 (2017) 583-587.

[6] TowerJazz (http://www.jazzsemi.com).

[7] S.Kushpil, F.Krizek, A.Isakov (ALICE Collaboration) IEEE Transactions on Nuclear Science V.66 I.11 (2019).

[8] Eudet-Memo-2010-12 EUTelescope (http://www.eudet.org/e26/).

[9] F. Krizek et al., Nucl. Instrum. Meth. A894 (2018) 87-95.

[10] Miljenko Suljić CERN-THESIS-2017-304. 